\title{
Distribución espacial del langostino patagónico (Pleoticus muelleri, (Bate, 1888)) y su relación con las variables ambientales, Golfo San Jorge, Argentina
}

\author{
Spatial distribution of the red shrimp (Pleoticus muelleri, (Bate, 1888)) and its relationship \\ with the environmental variables, San Jorge Gulf, Argentina \\ Mónica Fernández ${ }^{1}$, Daniel Hernández ${ }^{1}$ y Ana Roux ${ }^{1}$ \\ ${ }^{1}$ Instituto Nacional de Investigación y Desarrollo Pesquero (INIDEP). \\ Paseo Victoria Ocampo 1 B7602HSA, Mar del Plata, Argentina \\ lango@inidep.edu.ar
}

\begin{abstract}
The analysis of the distribution of shrimp concentrations in the south of the San Jorge Gulf is presented, as well as their relationship with the physico-chemical characteristics of the superficial sediments and the bottom water. Data analyzed were obtained from the Shrimp Project Research Cruise OB-05/03 carried out during June 2003. Biological variables analyzed were: juvenile, subadult and adult shrimp densities and size structure. Correspondence analyses were applied for numerical data treatment.

The distribution of the different shrimp categories in the studied area shows a west-eastern pattern, being the juveniles towards the west coast and the adults larger than $41 \mathrm{~mm}$ carapace length towards the northeast, while the main concentrations of subadults and adults were found in the rest of the study area. A relationship between the spatial distribution of the juvenile and adult categories and depth and total organic carbon (TOC) was determined. The highest adult densities (> $41 \mathrm{~mm}$ carcass length) were related with the highest depths and the lowest values of TOC. While the highest juvenile densities were related with the lowest depths and the highest TOC concentrations.
\end{abstract}

Key words: Shrimp fishery, Patagonia, spatial distribution, environmental variables

\section{Introducción}

Dentro de la plataforma continental argentina, el Golfo San Jorge es de gran importancia económica por ser caladero de especies de interés comercial entre las que se destacan el langostino Pleoticus muelleri (Bate, 1888) (Bertuche et al. 1999, 2000) y la merluza común Merluccius hubbsi Marini, 1933 (Aubone et al. 2000).
Resumen.- Se presenta el análisis de la distribución de las concentraciones de langostino en el sector sur del Golfo San Jorge y su vinculación con las características físico-químicas de los sedimentos superficiales y del agua de fondo. Los datos analizados provienen de la Campaña de Investigación del Proyecto Langostino OB-05/03 realizada en junio de 2003. Se analizaron las variables biológicas: densidad de individuos juveniles, subadultos y adultos de langostino, como así también la estructura de talla de los mismos. Para el tratamiento numérico de los datos se aplicaron análisis de correspondencia.

La distribución de las categorías de langostino en el área de estudio siguió un patrón oeste-este, localizándose los individuos juveniles hacia el litoral oeste y los adultos mayores de $41 \mathrm{~mm}$ de largo de caparazón, hacia el noreste, registrándose en el resto del área las principales concentraciones de subadultos y adultos. A la vez, se encontró una vinculación entre la distribución espacial de las categorías juveniles y adultos con las variables profundidad y concentración de carbono orgánico total en sedimentos (COT). Las mayores densidades de adultos $(>41 \mathrm{~mm}$ de largo de caparazón) se relacionaron con las mayores profundidades y los menores valores de COT. Mientras que las mayores densidades de juveniles se relacionaron con las menores profundidades y las mayores concentraciones de COT.

Palabras clave: Pesquería de langostino, Patagonia, distribución espacial, variables ambientales
El área sur comprendida entre las latitudes 46030’S y $47^{\circ} 00^{\prime} \mathrm{S}$ y el meridiano $66^{\circ} 00^{\prime} \mathrm{W}$ y la costa es de particular importancia en relación al recurso langostino ya que en la misma se localizan las mayores concentraciones que sustentan a la pesquería del litoral patagónico. Esta pesquería es una de las más importantes de Argentina resultando de particular importancia tanto para el mercado interno como para el 
externo. La pesquería tiene su inicio a principios de la década del 80 con un desembarco de 2616 t, y alcanza en el año 2000 las 36822 t, siendo las exportaciones de ese año cercanas a los 250 millones de dólares. En el año 2001 el desembarco declarado alcanzó un record histórico de 78797 t (SAGPyA 2001).

Dada la vinculación existente entre el sistema bentónico y el recurso langostino en sus diferentes etapas de su ciclo de vida, a partir del año 1992 se iniciaron diversos estudios orientados al conocimiento de los factores físico-químicos del agua, de los sedimentos y de las comunidades bentónicas en los fondos de distribución del langostino (Fernández \& Bertuche 1995, Roux et al. 1995, Roux \& Fernández 1997).

Es un hecho ampliamente aceptado que el sustrato constituye uno de los reguladores fundamentales de la distribución de los organismos bentónicos, habiéndose establecido fuertes correlaciones entre el tipo de sustrato y las asociaciones biológicas que en él se desarrollan (Sanders 1958, Buchanan et al. 1978, Eleftheriou \& Basford 1989, Etter \& Grassle 1992, Roux et al. 1995, Seiderer \& Newell 1999). Asimismo, en diversas pesquerías de peneidos se han identificado relaciones entre los índices de abundancia de las especies con ciertas características ambientales (Williams 1958, Loesch 1965, Grady 1971, Branford 1981, Jones 1987, Somers 1987).

En este estudio se describe el patrón de distribución del langostino y su vinculación con las variables ambientales durante junio de 2003 en el área sur del Golfo San Jorge, Argentina. Estas investigaciones tienen como principal objetivo contribuir en la definición de acciones relacionadas con el manejo y las estrategias de conservación de los recursos pesqueros.

\section{Material y métodos}

Los datos y las muestras provienen de la Campaña de Investigación del BIP Capitán Oca Balda del INIDEP OB-05/03 llevada a cabo en otoño-invierno de 2003 (9 al 29 de junio) en el Golfo San Jorge, Argentina. (Fig. 1).

Las muestras de langostino se obtuvieron de la captura de los 46 lances de pesca efectuados. Los lances se realizaron con una red de arrastre de fondo tipo tangonera cuyas características se describen en Fischbach (2003). Los muestreos de langostino

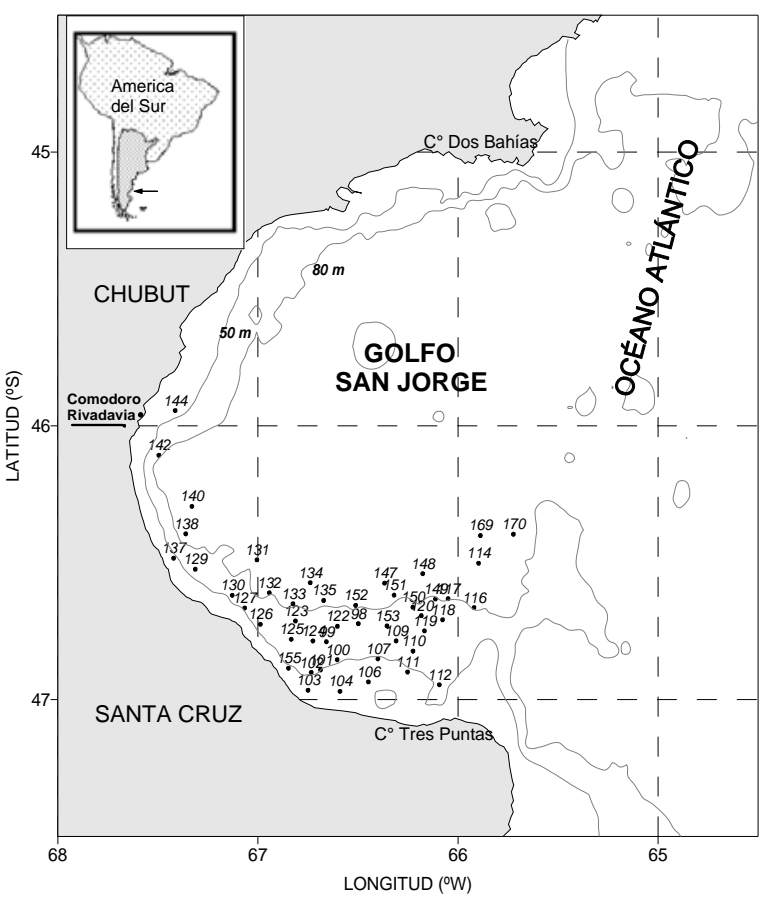

Figura 1

\section{Ubicación de las estaciones de muestreo}

Location of sampling stations

consistieron en la separación de los ejemplares por sexo, midiendo el largo de caparazón (Lcap) en mm. También se consignó el estado de madurez sexual, siguiendo los métodos de Angelescu \& Boschi (1959).

Para cada lance de pesca sobre la base de la información de la muestra (número de individuos por clase de talla y sexo y peso de la muestra) y las millas náuticas navegadas (Fischbach 2003), se calcularon las siguientes variables biológicas:

1) densidad del total de individuos en miles de individuos $\mathrm{mn}^{-2}$.

2) densidad de las siguientes categorías o rangos de tallas (Boschi 1989, Bertuche et al. 2000) en miles de individuos $\mathrm{mn}^{-2}$ :

- Individuos entre 4 y $24 \mathrm{~mm}$ de Lcap: individuos juveniles (pre-reclutas),

- Individuos entre 25 y $30 \mathrm{~mm}$ Lcap: individuos subadultos, 
- Individuos entre 31 y 40 mm Lcap: individuos adultos,

- Individuos mayores de $41 \mathrm{~mm}$ Lcap: individuos adultos mayores de $41 \mathrm{~mm}$.

3) Talla media del total de individuos y por sexo.

El análisis de los datos consistió en una ordenación de las variables en base a una matriz de datos estaciones x categorías de langostino. La ordenación espacial de las variables biológicas en cada muestra fue efectuada utilizando el análisis de correspondencia (AC) (Fernández 2006). Las muestras estudiadas para cada una de las 46 estaciones de muestreo se compararon en base a los datos de densidad estandarizados de las categorías citadas.

Para analizar las vinculaciones de los organismos con los parámetros físico-químicos, se utilizó el análisis de correspondencia canónica (ACC). Este análisis se efectuó considerando: 1) las variables ambientales: profundidad, temperatura, salinidad, concentración de oxígeno y clorofila $a$ en agua de fondo, porcentaje de limo, arcilla, carbono orgánico total, relación C: $\mathrm{N}$ en sedimentos y 2) las variables biológicas: densidad en número de individuos juveniles, individuos subadultos, individuos adultos e individuos adultos $>41 \mathrm{~mm}$ Lcap, aplicando una transformación Log $(x+1)$ de los datos (Ter Braak 1986, Ter Braak \& Smilauer 1998). La información correspondiente a las variables físicoquímicas de cada estación de muestreo se obtuvo a partir de los análisis realizados por Fernández et al. (2007).

Los análisis estadísticos se realizaron mediante el programa STATISTICA versión 6.0 (Stat Soft Inc. 1988) y el programa CANOCO versión 4.0 (Ter Braak \& Smilauer 1998).

\section{Resultados}

La estructura de talla del total de individuos y por sexo de la fracción estimada de abundancia relativa de langostino en el área, puede observarse en la Fig. 2.

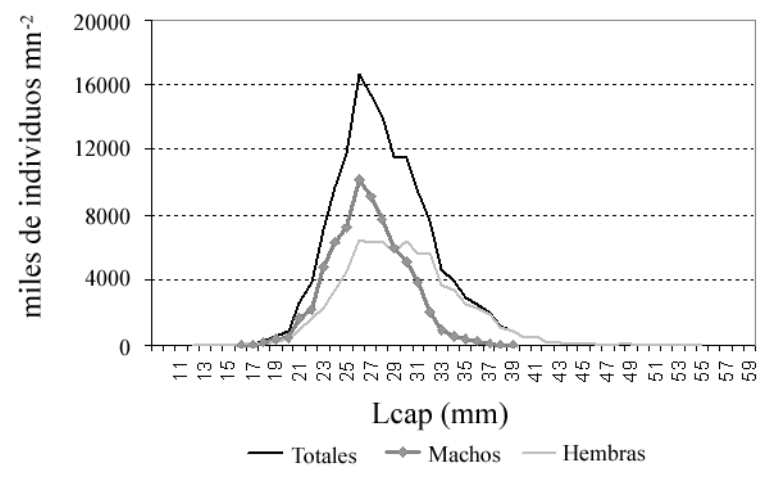

Figura 2

Estructura de talla del total de individuos y por sexo de $\boldsymbol{P}$. muelleri en el sector sur del Golfo San Jorge

Size structure of $P$. muelleri, total and by sex in the southern sector of the San Jorge Gulf

El rango de tallas para el total de individuos osciló entre $13 \mathrm{~mm}$ y $55 \mathrm{~mm}$ Lcap, con una talla media de 29,69 mm Lcap. El rango de tallas para machos y hembras osciló entre $18 \mathrm{~mm}$ y $39 \mathrm{~mm}$ Lcap y $13 \mathrm{~mm}$ y $55 \mathrm{~mm}$ Lcap, respectivamente, con una talla media de 28,27 mm para los machos y 31,03 mm Lcap para las hembras. La estructura de talla de los machos maduros se ubicó entre $28 \mathrm{~mm}$ y $39 \mathrm{~mm}$ de Lcap, con una talla media de 33,03 mm Lcap.

Considerando los valores de talla media para el total de individuos por lance, los mismos fluctuaron entre 23,20 mm y $38 \mathrm{~mm}$ Lcap, concentrándose los valores inferiores en el sur (23,50 mm Lcap) y en el sector costero oeste (26,76 mm a 29,52 mm Lcap), mientras que los superiores se encontraron en el este del área de estudio (30,13 mm a 32,79 mm Lcap).

La mayor participación en número se concentra en la categoría que agrupa a los individuos subadultos seguida por la categoría de adultos, con un 55,58\% y $32,50 \%$, respectivamente (Tabla 1). Los juveniles y adultos mayores de $41 \mathrm{~mm}$ Lcap presentaron valores en número de $10,72 \%$ y 1,21\%, respectivamente.

La abundancia relativa media de langostino para el área de estudio, fue de 142.401 miles de individuos $\mathrm{mn}^{-2}$. 
Tabla 1

Número de individuos de $P$. muelleri por categorías, porcentaje de hembras (H) y machos (M), talla media (Lcap en mm) y desviación estándar (DE) del total de individuos y por sexo, de cada estación de muestro. Campaña de Investigación OB-05/03 (Invierno de 2003)

Number of $P$. muelleri specimens by categories, females $(\mathrm{H})$ and males $(\mathrm{M})$ percentages, mean size (Lcap en mm) and standard deviation (DE) of the total specimens and by sex, from each sampling station. Cruise OB-05/03 (Winter 2003)

\begin{tabular}{|c|c|c|c|c|c|c|c|c|c|c|}
\hline $\begin{array}{c}\text { Estación } \\
\text { general }\end{array}$ & $\begin{array}{c}\begin{array}{c}\text { Juveniles } \\
\text { (miles de }\end{array} \\
\text { indiv. } \mathbf{m n}^{-2} \text { ) }\end{array}$ & $\begin{array}{c}\text { Subadultos } \\
\text { (miles de } \\
\text { indiv. } \mathbf{m n}^{-2} \text { ) }\end{array}$ & $\begin{array}{c}\text { Adultos } \\
\text { (miles de } \\
\text { indiv. } \mathbf{m n}^{-2} \text { ) }\end{array}$ & $\begin{array}{c}\text { Adul. }>\text { 41mm } \\
\text { (miles de } \\
\text { indiv. } \mathrm{mn}^{-2} \text { ) }\end{array}$ & $\begin{array}{c}\text { Total } \\
\text { (miles de } \\
\text { indiv. } \mathbf{m n}^{-2} \text { ) }\end{array}$ & $\begin{array}{c}\mathbf{H} \\
(\%)\end{array}$ & $\begin{array}{c}\text { M } \\
(\%)\end{array}$ & $\begin{array}{c}\text { Talla } \\
\text { media } \\
(\mathbf{m m})\end{array}$ & $\begin{array}{c}\text { Talla } \\
\text { media } \\
\text { Machos } \\
\text { (mm) }\end{array}$ & $\begin{array}{c}\text { Talla } \\
\text { media } \\
\text { Hembras } \\
(\mathbf{m m})\end{array}$ \\
\hline 98 & 644 & 1,700 & 868 & 33 & 3.245 & 54,18 & 45,82 & 28,92 & 27,58 & 30,05 \\
\hline 99 & 4.293 & 16.265 & 6.777 & 0 & 27.335 & 54,82 & 45,18 & 28,46 & 27,20 & 29,50 \\
\hline 100 & 78 & 246 & 129 & 3 & 456 & 52,63 & 47,37 & 28,86 & 28,21 & 29,45 \\
\hline 102 & 39 & 198 & 69 & 3 & 309 & 57,28 & 42,72 & 28,99 & 27,23 & 30,30 \\
\hline 103 & 6 & 21 & 15 & 0 & 42 & 57,14 & 42,86 & 30,00 & 29,50 & 30,38 \\
\hline 104 & 3 & 0 & 0 & 0 & 3 & 100,0 & - & 23,50 & 23,50 & - \\
\hline 106 & 0 & 21 & 18 & 0 & 39 & 46,15 & 53,85 & 30,58 & 29,50 & 31,83 \\
\hline 107 & 190 & 1.609 & 893 & 27 & 2.719 & 52,19 & 47,81 & 29,72 & 28,27 & 31,05 \\
\hline 109 & 112 & 885 & 572 & 45 & 1.614 & 61,59 & 38,41 & 30,32 & 28,18 & 31,64 \\
\hline 110 & 158 & 1.080 & 519 & 25 & 1.782 & 58,08 & 41,92 & 29,66 & 28,14 & 30,74 \\
\hline 112 & 11 & 218 & 178 & 7 & 414 & 61,35 & 38,65 & 30,86 & 28,83 & 32,13 \\
\hline 114 & 20 & 393 & 284 & 4 & 701 & 49,36 & 50,64 & 30,58 & 29,30 & 31,90 \\
\hline 116 & 196 & 2.437 & 2.117 & 107 & 4.857 & 49,82 & 50,18 & 31,07 & 29,45 & 32,71 \\
\hline 117 & 28 & 376 & 140 & 0 & 544 & 44,85 & 55,15 & 29,51 & 28,34 & 30,96 \\
\hline 118 & 442 & 3.214 & 1.903 & 91 & 5.650 & 53,58 & 46,42 & 30,13 & 28,46 & 31,58 \\
\hline 119 & 852 & 4.262 & 1.858 & 132 & 7.104 & 51,70 & 48,30 & 29,37 & 27,81 & 30,84 \\
\hline 120 & 60 & 484 & 675 & 65 & 1.284 & 53,74 & 46,26 & 2,32 & 30,00 & 34,30 \\
\hline 122 & 81 & 605 & 410 & 18 & 1.114 & 60,14 & 39,86 & 30,16 & 28,38 & 31,35 \\
\hline 123 & 285 & 917 & 524 & 6 & 1.732 & 56,06 & 43,94 & 29,10 & 27,34 & 30,47 \\
\hline 124 & 471 & 2.244 & 1.082 & 12 & 3.809 & 53,74 & 46,26 & 29,11 & 27,69 & 30,33 \\
\hline 125 & 99 & 1.850 & 1.175 & 14 & 3.138 & 43,59 & 56,41 & 30,40 & 29,28 & 31,85 \\
\hline 126 & 338 & 3.869 & 2.739 & 100 & 7.046 & 45,10 & 54,90 & 30,59 & 29,46 & 31,96 \\
\hline 127 & 1.065 & 3.808 & 1.227 & 18 & 6.118 & 53,99 & 46,01 & 28,21 & 26,60 & 29,58 \\
\hline 129 & 579 & 1.081 & 175 & 0 & 1.835 & 48,45 & 51,55 & 26,76 & 26,08 & 27,48 \\
\hline 132 & 707 & 1.928 & 370 & 16 & 3.021 & 49,26 & 50,74 & 27,45 & 26,27 & 28,65 \\
\hline 133 & 31 & 235 & 122 & 0 & 388 & 56,44 & 43,56 & 29,63 & 28,60 & 30,41 \\
\hline 134 & 76 & 794 & 405 & 4 & 1.279 & 50,43 & 49,57 & 29,84 & 28,50 & 31,17 \\
\hline 135 & 16 & 192 & 116 & 0 & 324 & 54,32 & 45,68 & 30,12 & 28,69 & 31,32 \\
\hline 138 & 88 & 526 & 108 & 0 & 722 & 49,17 & 50,83 & 28,30 & 27,93 & 28,67 \\
\hline 140 & 25 & 76 & 31 & 0 & 132 & 41,67 & 58,33 & 28,61 & 28,74 & 28,46 \\
\hline 142 & 24 & 110 & 18 & 0 & 152 & 51,32 & 48,68 & 27,95 & 26,60 & 29,19 \\
\hline 144 & 0 & 0 & 2 & 0 & 2 & 100,0 & 0,00 & 38,00 & 38,00 & - \\
\hline 147 & 20 & 1.024 & 1.604 & 68 & 2.716 & 55,89 & 44,11 & 32,53 & 30,20 & 34,37 \\
\hline 148 & 5 & 782 & 1.125 & 30 & 1.942 & 48,46 & 51,54 & 32,36 & 30,62 & 34,21 \\
\hline 149 & 191 & 2.295 & 1.736 & 44 & 4.266 & 51,05 & 48,95 & 30,69 & 29,26 & 32,05 \\
\hline 150 & 354 & 2.532 & 1.600 & 104 & 4.590 & 43,14 & 56,86 & 30,17 & 29,05 & 31,65 \\
\hline 151 & 80 & 1.677 & 4.073 & 280 & 6.110 & 50,03 & 49,97 & 33,40 & 30,92 & 35,89 \\
\hline 152 & 439 & 1.791 & 639 & 27 & 2.896 & 50,62 & 49,38 & 28,75 & 27,66 & 29,81 \\
\hline 153 & 191 & 1.032 & 312 & 13 & 1.548 & 48,71 & 51,29 & 28,56 & 27,40 & 29,78 \\
\hline 155 & 766 & 3.782 & 1.039 & 17 & 5.604 & 50,79 & 49,21 & 28,20 & 26,90 & 29,46 \\
\hline 169 & 24 & 1.277 & 2.793 & 74 & 4.168 & 47,05 & 52,95 & 32,79 & 31,19 & 34,59 \\
\hline 170 & 676 & 6.592 & 4.026 & 321 & 11.615 & 48,49 & 51,51 & 30,51 & 28,82 & 32,31 \\
\hline
\end{tabular}


Dadas las características de la estación 99, la misma se analiza de forma independiente. Esta estación concentra el máximo valor de abundancia de 27.335 miles de individuos $\mathrm{mn}^{-2}$ como así también de individuos juveniles, subadultos y adultos, que alcanzan los 4.293, 16.265 y 6.777 miles de individuos $\mathrm{mn}^{-2}$.

Considerando las restantes estaciones en conjunto, la mayor abundancia de langostino se localiza en los sectores este, con valores entre 4.266 y 11.615 miles de individuos $\mathrm{mn}^{-2}$ y costero sudoeste con valores entre 3.138 y 7.066 miles de individuos $\mathrm{mn}^{-2}$. En relación a la abundancia de las categorías analizadas, los mayores valores de individuos juveniles se localizan en el sector costero oeste (707 y 1.302 miles de individuos $\mathrm{mn}^{-2}$ ), de los individuos subadultos en los sectores este (4.266 y 11.615 miles de individuos $\mathrm{mn}^{-2}$ ) y costero sudoeste (3.138 y 7.066 miles de individuos $\mathrm{mn}^{2-}$ ) y de los individuos adultos (31-40 mm Lcap), al igual que la categoría anterior, en los sectores este (2.117 y 4.026 miles de individuos $\mathrm{mn}^{-2}$ ) y costero sudoeste (1.020 y 2.739 miles de individuos $\mathrm{mn}^{-2}$ ); mientras que las mayores abundancias de los adultos mayores de $41 \mathrm{~mm}$ Lcap, se ubicaron en el sector este con valores entre 104 y 321 miles de individuos $\mathrm{mn}^{-2}$.
Con respecto al análisis de correspondencia, la Tabla 2 muestra los autovalores, la inercia y la inercia acumulada correspondiente a cada dimensión. La primera dimensión acumula un 86,66\% de la inercia total y por lo tanto es la que tiene la mayor capacidad explicativa de los datos. El hecho que la primera dimensión concentre casi el total de la inercia debe interpretarse como consecuencia del patrón de distribución de los datos que responde a un gradiente de los valores de abundancia de las categorías analizadas en una dirección. En la Fig. 3 aparece la disposición de las categorías y las estaciones respecto a las dimensiones 1 y 2. En la representación se observa

\section{Tabla 2}

Autovalores, inercia e inercia acumulada para las tres primeras dimensiones del análisis de correspondencia

Autovalues, inertia and accumulated inertia for the first three dimensions of the correspondence analysis

\begin{tabular}{lrcc}
\hline & autovalor & $\begin{array}{c}\text { Inercia } \\
\text { \% }\end{array}$ & $\begin{array}{c}\text { Inercia } \\
\text { acumulada (\%) }\end{array}$ \\
\hline Dimensión 1 & 0,110 & 86,658 & 86,658 \\
Dimensión 2 & 0,012 & 9,190 & 95,849 \\
Dimensión 3 & 0,005 & 4,151 & 100,000 \\
\hline
\end{tabular}

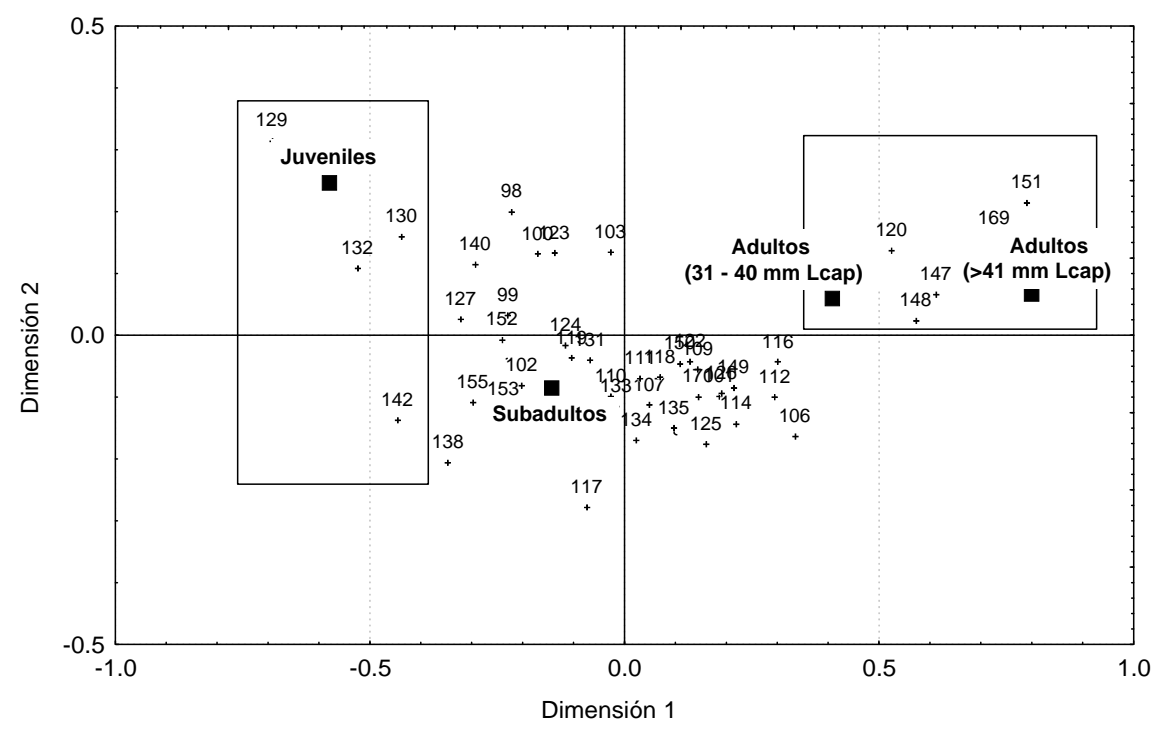

Figura 3

Análisis de correspondencia entre las categorías de $P$. muelleri y las estaciones de muestreo, en el sector sur del Golfo San Jorge

Correspondence analysis among $P$. muelleri categories and sampling stations in the southern sector of the San Jorge Gulf 
que dentro del gradiente de variación indicado por las dimensiones 1 y 2 en el plano definido por el extremo positivo del primer eje se localizan los individuos adultos mayores a $41 \mathrm{~mm}$ Lcap y adultos con tallas entre 31 y $40 \mathrm{~mm}$ Lcap presentes en las estaciones ubicadas en el sector noreste del golfo, claramente separadas de los individuos juveniles (en la parte negativa del primer eje) que han aparecido en estaciones ubicadas en el sector costero oeste, diferenciándose entre los juveniles y adultos, los individuos subadultos presentes en las estaciones ubicadas en el resto del área de estudio (sector central y sudeste).

De manera general, la distribución de las categorías de langostino indica una distribución espacial de las estaciones desde el sector noreste (1) hacia el sector costero oeste (2) pasando por el sector central y sudeste (3), que corresponden a tres sectores con características particulares.

El primero se caracteriza por presentar:

- el mayor valor medio de densidad de individuos adultos entre $31 \mathrm{~mm}$ y $40 \mathrm{~mm}$ Lcap (2.054,00 miles de individuos $\mathrm{mn}^{-2}$ ) y de adultos mayores de $41 \mathrm{~mm}$ Lcap (103,00 miles de individuos $\mathrm{mn}^{-2}$ ),

- el menor valor medio de densidad de juveniles (37,80 miles de individuos $\mathrm{mn}^{-2}$ ),

- el mayor valor promedio de talla media $(30,86 \mathrm{~mm}$ Lcap).

El segundo, se caracteriza por presentar:

- el mayor valor medio de abundancia de individuos juveniles $\left(653,00\right.$ miles de individuos $\mathrm{mn}^{-2}$ ),

- el menor valor medio de abundancia de individuos adultos mayores de $41 \mathrm{~mm}$ Lcap (4,00 miles de individuos $\mathrm{mn}^{-2}$ ) y de individuos adultos entre 31 y 40 mm Lcap (395,75 miles de individuos $\mathrm{mn}^{-2}$ ),

- el menor valor promedio de talla media $(27,29 \mathrm{~mm}$ Lcap);

El tercer sector que comprende el área definida entre los dos primeros sectores, se caracteriza por presentar:

- el mayor valor medio de abundancia de individuos subadultos $\left(1.924,06\right.$ miles de individuos $\left.\mathrm{mn}^{-2}\right)$ más allá de los valores mínimos y máximos de densidad total (39,00 y 27.656,00 miles de individuos $\mathrm{mn}^{-2}$ ),

- valores intermedios de los valores promedio de abundancia de individuos juveniles (355,54 miles de individuos $\mathrm{mn}^{-2}$ ) como así también de adultos entre 31 y $40 \mathrm{~mm}$ Lcap (982,40 miles de individuos $\mathrm{mn}^{-2}$ ) y adultos mayores a $41 \mathrm{~mm}$ Lcap (33,97 miles de individuos $\mathrm{mn}^{-2}$ ).

- un valor promedio de talla media de 29,67 mm Lcap, valor intermedio a los observados para el sector 1 y 2.

En relación al análisis de correspondencia canónica, la prueba de permutación (500 permutaciones) permitió establecer la significación estadística del primer eje canónico ( $F=16.984 ; P<0,05)$. Las correlaciones canónicas entre las variables biológicas y las variables ambientales son: 0,674; 0,292; 0,244 y 0,000. El primer eje permite explicar el 32,1\% de la variación de las variables biológicas y el 93,1 \% de la variación correspondiente a la variación de la relación entre las variables biológicas y las ambientales. El primer eje canónico describe básicamente los cambios en COT, arcilla y profundidad. En la Tabla 3 se presenta la correlación entre el primer eje canónico obtenido a partir de las variables ambientales y cada una de las variables ambientales consideradas.

\section{Tabla 3}

\section{Correlación entre el primer eje canónico obtenido a partir de las variables ambientales y cada una de las variables ambientales consideradas}

Correlation among the first canonical axis obtained from environmental variables and each considered environmental variables

\begin{tabular}{cc}
\hline Variables & Correlación \\
\hline Limo & 0,2739 \\
Arcilla & $-0,4267$ \\
COT & $-0,8886$ \\
Relación C:N & $-0,1472$ \\
Profundidad & 0,4456 \\
Temperatura & $-0,1179$ \\
Salinidad & 0,0976 \\
Concentración Oxígeno & $-0,1797$ \\
Clorofila $a$ (AF) & $-0,1166$ \\
\hline
\end{tabular}




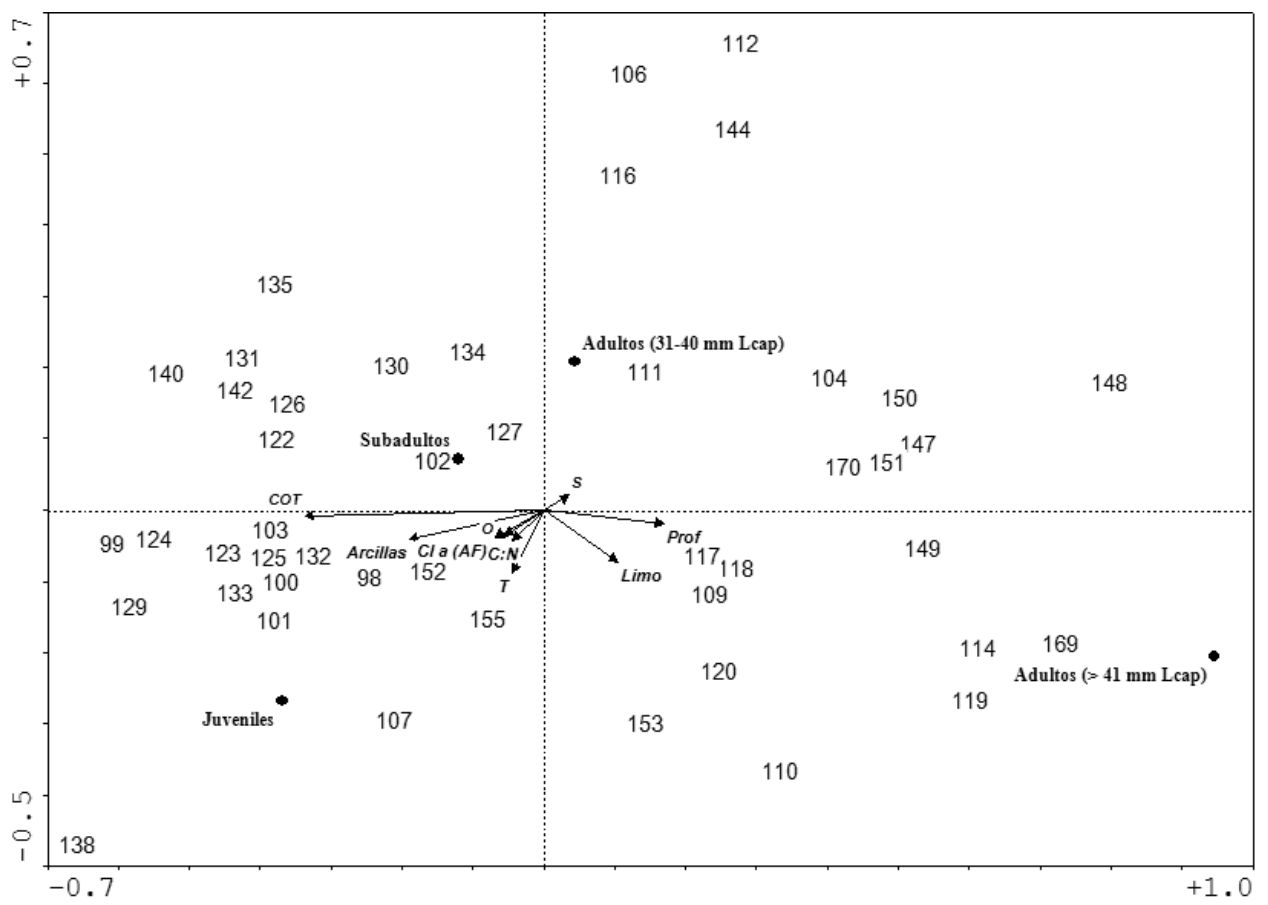

Figura 4

Diagrama de ordenación de las variables biológicas, ambientales y de las estaciones

Triplot of the biological and environmental variables and stations

En la Fig. 4 se puede ver el diagrama de ordenación de las variables biológicas, ambientales y de las estaciones. Como puede observarse, los juveniles están asociados con altos valores de COT y profundidades bajas, y los adultos (> $41 \mathrm{~mm}$ Lcap) con altas profundidades y bajos valores de COT. La correlación entre la profundidad y el COT es igual a 0,2009 y es estadísticamente no significativa $(P=0,1807)$, de esta forma la preferencia de los juveniles y adultos depende la profundidad y de la COT, conjuntamente.

\section{Discusión}

La densidad media relativa de las concentraciones de langostino del sur del Golfo San Jorge durante el invierno de 2003, como así también la composición porcentual en número estimada en el área, en términos de las categorías de estudio, concuerdan con los valores obtenidos en los estudios de evaluación del recurso langostino realizados por Fischbach (2003) en junio de 2003 en la totalidad del Golfo San Jorge, que indican para el área sur del golfo una densidad media relativa de langostino de 101.583 miles de individuos $\mathrm{mn}^{-2}$ según una estimación por el método de área barrida, localizándose las concentraciones máximas tanto en el sector costero oeste como en el noreste. Según este autor, la mayor composición porcentual en número de la biomasa estimada corresponde a individuos de largo de caparazón entre 24 y 34 mm, individuos principalmente subadultos.

De manera general, se observa una disposición de las categorías de langostino en el área siguiendo un patrón oeste-este en el golfo, localizándose los individuos juveniles hacia el litoral oeste y los adultos en máximas densidades hacia el noreste, distribuyéndose en el resto del área las principales concentraciones de subadultos y adultos, categorías predominantes de langostino durante este período de estudio, como ya fue mencionado anteriormente. En relación a las mayores concentraciones de individuos adultos localizados en el noreste del área de estudio, se observa coincidencia con los resultados obtenidos por De la Garza \& Fischbach (2000, 2002) en sus estudios de distribución espaciotemporal de concentraciones de langostino patagónico y con los resultados del programa de marcación y 
recaptura del langostino marcado, implementado por el INIDEP, desde el año 2000 (Piñero \& Roux 2000, Piñero et al. 2001, 2002, 2003, 2004).

De la Garza \& Fischbach (2000) en sus estudios realizados en relación a la producción de la flota tangonera en el primer semestre del año 2000 en el Golfo San Jorge, indican que los individuos entre 30 mm y 38 mm Lcap se localizaron en la boca del golfo y en aguas al noreste del mismo, y en particular durante el mes de junio en el sur de la boca del golfo ( $46^{\circ} 00^{\prime} \mathrm{S}$

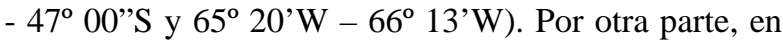
sus estudios del segundo semestre de los años 1998, 2000 y 2001, estos autores encontraron que la tendencia de las tallas durante todos los meses analizados presentó un comportamiento homogéneo, localizándose las tallas más grandes (> 37 mm Lcap para machos y $44 \mathrm{~mm}$ Lcap para hembras) hacia el norte-noreste y en aguas exteriores del Golfo San Jorge (boca del golfo); por su parte, las tallas menores $(<36$ mm Lcap y 43 mm Lcap, para machos y hembras, respectivamente) lo hicieron en las aguas interiores del golfo (De la Garza \& Fischbach 2002). Piñero \& Roux (2000) y Piñero et al. (2001, 2002, 2003, 2004) en sus estudios de marcación y recaptura del langostino marcado en el Golfo San Jorge, indican un desplazamiento de las agregaciones de langostino adulto desde el área sur del golfo en sentido principalmente este-noreste. Los autores antes mencionados relacionan este desplazamiento con la dinámica propia del recurso.

Considerando la vinculación entre la distribución y abundancia de las categorías de langostino con las variables físico-químicas del agua de fondo y de los sedimentos, se encontraron relaciones sólo entre las categorías juveniles y adultos (> $41 \mathrm{~mm}$ Lcap) y las variables profundidad y concentración de carbono orgánico total en sedimentos. Los juveniles se localizaron en zonas costeras de baja profundidad, granulometría fina y alto contenido de COT; mientras que los adultos, en el noreste del área de estudio, en zonas de mayores profundidades, granulometría mas gruesa y bajo contenido de COT. Según lo indicado para las pesquerías de peneidos del Golfo de México (Williams 1958, Grady 1971), de la bahía de Mobile, Alabama, EUA (Loesch 1965), de la costa Sudanesa del Mar Rojo (Branford 1981) y del Golfo de Carpentaria, Australia (Somers 1987), la profundidad y la granulometría fina se consideran de importancia desde el punto de vista de protección y refugio, mientras que el contenido de COT, como medida indirecta de la disponibilidad de alimento.

Williams (1958) estudió, de manera experimental, la relación entre la distribución de Penaeus setiferus, $P$. aztecus y $P$. duorarum con cinco tipos de sustrato. Los resultados de sus experimentos indicaron que la distribución de los peneidos en los sustratos no es casual. $P$. duorarum se relacionó con el sustrato constituido por arena, mientras que $P$. aztecus y $P$. setiferus con aquellos más fangosos. Según este autor, tanto el tipo sustrato como el contenido alimenticio de las partículas fueron los factores que condicionaron la distribución de las especies.

Loesch (1965), analizó la distribución y crecimiento de los organismos juveniles de los peneidos Penaeus fluviatilis, $P$. aztecus y $P$. duorarum de la bahía de Mobile, Alabama, EUA; llegando a la conclusión que la mayor abundancia se ubicó en aguas de baja profundidad $(0,60 \mathrm{~m}-1 \mathrm{~m})$ con vegetación y grandes cantidades de detrito orgánico.

Branford (1981) estudió la distribución de Penaeus semisulcatus, $P$. latisulcatus y Metapenaeus monoceros, del Mar Rojo y observó que la mayor abundancia de los peneidos aumentaba a medida que lo hacia la finura de los sedimentos y el contenido de carbono orgánico, indicando que el tamaño de las partículas es importante para enterrarse y por el contenido de carbono orgánico.

Somers (1987) investigó sobre el tipo sedimentario, el contenido de carbono orgánico de los sedimentos y la profundidad como factores condicionantes de la distribución de las especies comerciales de peneidos del Golfo de Carpentaria (Penaeus esculentus, $P$. semisulcatus, Metapenaeus ensis, M. endeavouri, $P$. latisulcatus, $P$. longistylus, $P$. merguiensis $\mathrm{y}$ Solenocera australiana). La profundidad se considera como la causante de la mayor variación de la captura por unidad de esfuerzo. La distribución de la captura por unidad de esfuerzo de todas las especies comerciales fue relativamente uniforme y no mostró correlación con el tipo de sedimento, con el porcentaje de fango, ni con la concentración de carbono orgánico; sólo el 13 \% de la variación puede ser explicada por una preferencia del rango de profundidad. A diferencia de los adultos, los juveniles se hallaron en agua costeras $(<20 \mathrm{~m})$. Sin embargo, los experimentos de marcación realizados sobre la mayoría de las especies comerciales demostraron la preferencia de $P$. 
esculentus y $P$. semisulcatus por diferentes tipos de sedimentos.

En relación a la distribución de los peneidos y su vinculación con las variables hidrográficas, se destaca el estudio realizados por Ye et al. (1999). Estos autores realizaron un análisis de las preferencias ambientales de Penaeus semisulcatus, Metapenaeus affinis y Penaeus stylifera en aguas de Kuwait, encontrando que cada especie tiene distintas preferencias con la temperatura, salinidad y profundidad. La profundidad fue la variable determinante en la distribución de estos peneidos.

\section{Agradecimientos}

Los autores desean expresar su agradecimiento a los evaluadores por su importante aporte.

\section{Literatura citada}

Angelescu V \& E Boschi. 1959. Estudio biológico pesquero del langostino de Mar del Plata en conexión con la operación nivel medio. Servicio de Hidrografía Naval, Argentina Público H1017: 1-135.

Aubone A, S Bezzi, R Castrucci, C Dato, P Ibáñez, G Irusta, M Perez, M Renzi, B Santos, N Scarlato, M Simonazzi, L Tringalli \& F Villarino. 2000. Merluza (Merluccius hubbsi). En: Bezzi S, R Akselman \& E Boschi (eds), Síntesis del estado de las Pesquerías Marítimas Argentinas y de la Cuenca del Plata. Años 1997-1998, con la actualización de 1999, pp. 29-39. INIDEP, Mar del Plata.

Bertuche D, C Fischbach, A Roux \& M Fernández. 1999. Langostino (Pleoticus muelleri). En: Cajal J \& B Prenski (eds). Diagnóstico de los Recursos Pesqueros, pp. 110120. INIDEP, Mar del Plata.

Bertuche D, C Fischbach, A Roux, M Fernández \& R Piñero. 2000. Langostino (Pleoticus muelleri). En: Bezzi S, R Akselman \& E Boschi (eds), Síntesis del estado de las Pesquerías Marítimas Argentinas y de la Cuenca del Plata. Años 1997-1998, con la actualización de 1999, pp. 179-190. INIDEP, Mar del Plata.

Boschi EE. 1989. Biología pesquera del langostino del litoral patagónico de Argentina (Pleoticus muelleri). Contribuciones INIDEP 646: 1-71.

Branford JR. 1981. Sediment and the distribution of penaeid shrimp in the Sudanese Red Sea. Estuarine, Coastal and Shelf Science 13: 349-355.

Buchanan JB, M Sheader \& PF Kingston. 1978. Sources of variability in the benthic macrofauna off the south
Northumberland coast, 1971-1976. Journal of the Marine Biological Association of the United Kingdom 58: 191209.

De la Garza J \& $\mathbf{C}$ Fischbach. 2000. Langostino patagónico: la producción de la flota tangonera en el primer semestre del 2000. Informe Técnico Interno DNI INIDEP 22: 1-14.

De la Garza J \& C Fischbach. 2002. Distribución espaciotemporal y estructura de las talla del langostino patagónico durante los meses de agosto a noviembre de los años 1998, 2000 y 2001. Informe Técnico Interno DNI INIDEP 45: 1-14.

Eleftheriou A \& DJ Basford. 1989. The macrobenthic infauna of the offshore northern North Sea. Journal of the Marine Biological Association of the United Kingdom 69: 123-143.

Etter R \& J Grassle. 1992. Patterns of species diversity in the deep sea as a function of sediment particle size diversity. Nature 360: 576-578.

Fernández M. 2006. Características físico-químicas de los sedimentos del Golfo San Jorge y su relación con los organismos bentónicos del sector. Tesis Doctoral, Universidad Nacional de Mar del Plata, Argentina, 307 pp.

Fernández M \& D Bertuche. 1995. Relevamiento del estado de los sedimentos de los fondos de pesca de la pesquería de langostino patagónico. Años 1992 y 1993. Informe Técnico Interno DNI INIDEP 5: 1-16.

Fernández M, D Cucchi Colleoni, A Roux, A Marcos \& E Fernández. 2007. Caracterización físico-química del sistema bentónico en el sector sur del Golfo San Jorge, Argentina. Revista de Biología Marina y Oceanografía 42(2): 177-192.

Fischbach C. 2003. Informe de Campaña OB-05/03. Proyecto Langostino. Informe de Campaña. INIDEP, pp.1-18.

Grady JR. 1971. The distribution of sediment properties and shrimp catch on two shrimping grounds on the continental shelf of Mexico. Proceedings of Gulf and Caribbean Fisheries Institute 23: 39-48.

Jones M. 1987. Surficial sediments of western Gulf of Carpentaria, Australia. Australian Journal of Marine and Freshwater Research 38: 151-167.

Loesch L. 1965. Distribution and growth of penaeid shrimp in Mobile Bay, Alabama. Publications of the Institute of Marine Science, University of Texas 10: 41-58.

Piñero R \& A Roux. 2000. Plan de marcación de langostino patagónico. I. Resultados de la experiencia piloto de marcado y supervivencia (campaña de investigación OB03/00). Informe Técnico Interno DNI INIDEP 49: 1-12. 
Piñero R, A Roux \& J De la Garza. 2001. Plan de marcación de langostino patagónico. III. Inicio de la actividad de marcación y liberación de langostino patagónico (Pleoticus muelleri) en concentraciones del Golfo San Jorge, durante la campaña de investigación OB-06/01. Informe Técnico Interno DNI INIDEP 39: 112.

Piñero R, A Roux \& J De la Garza. 2002. Plan de marcación del langostino patagónico. Informe de avance y actualización de los datos de recaptura. Período setiembre de 2001 - julio de 2002. Informe Técnico Interno DNI INIDEP 73: 1-13.

Piñero R, A Roux \& J De la Garza. 2003. Plan de marcación de langostino patagónico. Informe de avance y actualización de los datos de recaptura. Período agosto diciembre 2002. Informe Técnico Interno DNI INIDEP 17: $1-5$.

Piñero R, A Roux \& J De la Garza. 2004. Plan de marcación de langostino patagónico. Actualización de los datos de recaptura: Período julio 2003 - febrero 2004 y reporte de las tareas de marcado y liberación desarrolladas durante la campaña OB - 01/04. Informe Técnico Interno DNI INIDEP 26: 1-8.

Roux A \& M Fernández. 1997. Caracterización de los fondos de pesca del langostino Pleoticus muelleri en el Golfo San Jorge y litoral de la provincia de Chubut. Argentina. Informe Técnico Interno INIDEP 13: 1-28.

Roux, A, M Fernández \& C Bremec. 1995. Preliminary survey of the benthic communities of patagonian shrimp fishing grounds in San Jorge Gulf (Argentina). Ciencias Marinas 21 (3): 295-310.
Roux A, R Piñero \& J De la Garza. (En prensa). Guía para la identificación de la fauna asociada a la pesquería del langostino patagónico. Publicaciones Especiales INIDEP.

Sanders H. 1958. Benthic studies in Buzzard Bay. I. Animalsediment relationship. Limnology and Oceanography 3 (3): 245-258.

SAGPyA. 2001. Capturas Marítimas Totales 2001. 8 pp. Secretaría de Agricultura, Ganadería, Pesca y Alimentación, Buenos Aires.

Seiderer I \& A Newell. 1999. Analysis of the relationship between sediment composition and benthic community structure in coastal deposits. ICES Journal of Marine Science 56: 757-765.

Somers I. 1987. Sediment type as a factor in the distribution of the commercial prawn species of the western Gulf of Carpentaria, Australia. Australian Journal of Marine and Freshwater Research 38: 133-149.

Stat Soft Inc.. 1998. Statistica Software, versión 6.0. Stat Soft, Tulsa.

Ter Braak C. 1986. Canonical correspondence analysis a new eigenvectors technique for multivariate direct gradient analysis. Ecology 67(5): 1167-1179.

Ter Braak C \& P Smilauer. 1998. CANOCO Reference Manual and User's Guide to Canoco for Windows: Software for Canonical Community Ordination (version 4), 352 pp. Microcomputer Power, Ithaca.

Williams A. 1958. Substrates as a factor in shrimp distribution. Limnology and Oceanography 3: 283-290.

Ye Y, H Mohammed \& J Bishop. 1999. Depth, temperature and salinity references of newly recruit penaeid shrimps in Kuwait waters. Fishery Oceanography 8 (2): 128-138. 